Kragujevac Journal of Mathematics

Volume 45(4) (2021), Pages 571-585.

\title{
FRACTIONAL ORDER OPERATIONAL MATRIX METHOD FOR SOLVING TWO-DIMENSIONAL NONLINEAR FRACTIONAL VOLTERRA INTEGRO-DIFFERENTIAL EQUATIONS
}

\author{
AMIRAHMAD KHAJEHNASIRI ${ }^{1}$, M. AFSHAR KERMANI ${ }^{1}$, AND REZZA EZZATI ${ }^{2 *}$
}

\begin{abstract}
This article presents a numerical method for solving nonlinear twodimensional fractional Volterra integral equation. We derive the Hat basis functions operational matrix of the fractional order integration and use it to solve the two-dimensional fractional Volterra integro-differential equations. The method is described and illustrated with numerical examples. Also, we give the error analysis.
\end{abstract}

\section{INTRODUCTION}

Fractional differential and integral equations involving the Caputo fractional operator or the Riemann-Liouville fractional operator has been paid more and more attention. There are several numerical methods for solving fractional integro-differential equations. Such as Haar wavelet method [24], CAS wavelets [25], Bernstein polynomials [1], collocation method [23], fractional differential transform method [3], Block pulse operational matrix $[20,28]$.

Integro-differential equation of fractional order has been proved to be valuable tools to model the dynamics of many processes in various fields of science and engineering through strongly anomalous media. Indeed, we can find numerous applications in electro-chemistry, viscoelasticity, signal processing, economies, electromagnetic, etc. $[9,10,18,22]$.

Hat functions (HFs) are a powerful mathematical tool for solving various kinds of equations. The solution of stochastic Ito-Volterra integral equations based on stochastic operational matrix [11], E. Babolian et al. have applied this method for

Key words and phrases. Hat basis functions, operational matrix, error analysis, block pulse function, two-dimensional fractional integral equation.

2010 Mathematics Subject Classification. Primary: 26A33. Secondary: 45G10, 65Gxx.

DOI $10.46793 / \mathrm{KgJMat} 2104.571 \mathrm{~K}$

Received: December 16, 2018.

Accepted: March 28, 2019. 
solving systems of nonlinear integral equations [5], M. H. Heydari et al. have applied Hat functions for solving nonlinear stochastic Ito integral equations [11,13]. F. Mirzaee and E. Hadadiyan have used two-dimensional Hat functions for solving space-time integral equations [17]. M. P. Tripathi et al. have applied HFs for solving fractional differential equations [27].

The operational matrix of integration has been determined for several types of orthogonal polynomials, such as Legendre polynomials [21], Laguerre series [12], and Block-pulse functions [4,7], Triangular functions [15]. The operational matrix of fractional derivatives has been determined for some types of orthogonal polynomials, such as Legendre polynomials [26], Chebyshev polynomials [6], Triangular functions $[8,14]$.

In this paper, two dimensional Hat functions (2DHFs) will be used to solve the following nonlinear two-dimensional fractional integral equation

$D_{x}^{\alpha} u(x, y)=f(x, y)+\frac{1}{\Gamma(\alpha) \Gamma(\beta)} \int_{0}^{y} \int_{0}^{x}(y-s)^{\alpha-1}(x-t)^{(\beta-1)} G(x, y, s, t, u(s, t)) d s d t$,

with the initial conditions

$$
\frac{\partial^{i}}{\partial x^{i}} u(0, y)=\delta_{i}, \quad i=0,1, \ldots, \rho-1, \rho-1<\alpha \leq \rho, \rho \in \mathbb{N},
$$

where $(\alpha, \beta) \in(0, \infty) \times(0, \infty), u \in L^{1}(\Omega), \Omega:=[0, a] \times[0, b]$, are known functions, (1.1) is the Caputo fractional differentiation operator and the unknown function $u(x, y)$ to be determined. In this work, we consider that, the nonlinear function has the following form $G(x, y, s, t, u)=k(x, y, s, t,)[u(s, t)]^{P}$, where $p$ is positive integer. In this paper, we introduce a new operational method to solve nonlinear two dimensional fractional Volterra integro-differential equations. The method is based on reducing the equation to the system of algebraic equation by expanding the solution as Hat functions.

\section{Riemann-Liouville and Caputo Fractional Derivatives}

There are various types of definition for the fractional derivative. The most commonly used definitions are Riemann-Liouville and Caputo formulas. RiemannLiouville fractional integration of order $\alpha$ is defined as

$$
I_{x_{0}}^{\alpha} f(x)=\frac{1}{\Gamma(\alpha)} \int_{x_{0}}^{x}(x-t)^{\alpha-1} f(t) d t, \quad \alpha>0, x>0 .
$$

The following equations define Riemann-Liouville and Caputo fractional derivatives of order $\alpha$, respectively,

$$
\begin{aligned}
D_{x_{0}}^{\alpha} f(x) & =\frac{d^{m}}{d x^{m}}\left[I_{x_{0}}^{m-\alpha} f(x)\right], \\
D_{* x_{0}}^{\alpha} f(x) & =I_{x_{0}}^{m-\alpha}\left[\frac{d^{m}}{d x^{m}} f(x)\right],
\end{aligned}
$$


where $m-1 \leq \alpha<m$ and $n \in \mathbb{N}$. From (2.1) and (2.2), we have

$$
D_{x_{0}}^{\alpha} f(x)=\frac{1}{\Gamma(m-\alpha)} \frac{d^{m}}{d x^{m}} \int_{x_{0}}^{x}(x-t)^{m-\alpha-1} f(t) d t, \quad x>x_{0} .
$$

Lemma 2.1. If $n-1<\alpha \leq n, n \in \mathbb{N}$, then $D_{x}^{\alpha} I^{\alpha} u(x, t)=u(x, t)$, and

$$
I^{\alpha} D_{x}^{\alpha} \mathrm{u}(x, t)=\mathrm{u}(x, t)-\sum_{k=0}^{n-1} \frac{\partial^{k} u\left(0^{+}, t\right)}{\partial x^{k}} \frac{x^{k}}{k !}, \quad x>0 .
$$

Definition $2.1([2])$. Let $(\alpha, \beta) \in(0, \infty) \times(0, \infty), \theta=(0,0), \Omega:=[0, a] \times[0, b]$, and $u \in L^{1}(\Omega)$. The left-sided mixed Riemann-Liouille integral of order $(\alpha, \beta)$ of $u$ is defined by

$$
\left(I_{\theta}^{(\alpha, \beta)} u\right)(x, y)=\frac{1}{\Gamma(\alpha) \Gamma(\beta)} \int_{0}^{y} \int_{0}^{x}(y-s)^{(\alpha-1)}(x-t)^{(\beta-1)} u(s, t) d s d t .
$$

In particular

1. $\left(I_{\theta}^{(\alpha, \beta)} u\right)(x, y)=u(x, y)$;

2. $\left(I_{\theta}^{(\alpha, \beta)} u\right)(x, y)=\int_{0}^{x} \int_{0}^{y} u(s, t) d t d s,(x, y) \in \Omega, \sigma=(1,1)$;

3. $\left(I_{\theta}^{(\alpha, \beta)} u\right)(x, 0)=\left(I_{\theta}^{(\alpha, \beta)}\right)(0, y)=0, x \in[0, a], y \in[0, b]$;

4. $I_{\theta}^{\alpha, \beta} x^{\lambda} y^{\omega}=\frac{\Gamma(1+\lambda) \times \Gamma(1+\omega)}{\Gamma(1+\lambda+\alpha) \times \Gamma(1+\omega+\beta)} x^{\lambda+\alpha} y^{\omega+\beta},(x, y) \in \Omega, \lambda, \omega \in(-1, \infty)$.

\section{Review of Hat Functions and Their Properties}

A set of HFs is usually defined on $[0,1]$ as:

$$
\begin{aligned}
& \phi_{0}(t)= \begin{cases}\frac{h-t}{h}, \quad 0 \leq t<h, \\
0, \quad \text { otherwise }\end{cases} \\
& \phi_{i}(t)= \begin{cases}\frac{t-(i-1) h}{h}, & (i-1) h \leq t<i h, \\
\frac{(i+1) h-t}{h}, & i h \leq t<(i+1) h, i=1,2, \ldots, n-1, \\
0, & \text { otherwise }\end{cases} \\
& \phi_{n}(t)= \begin{cases}\frac{t-(1-h)}{h}, & T-h \leq t<T, \\
0, & \text { otherwise }\end{cases}
\end{aligned}
$$

where $h=\frac{1}{n}$ and $n$ is an arbitrary positive integer. Indeed, the unit interval $[0,1]$ is divided into $n$ equidistant subintervals. According to the definition of HFs, we have

$$
\phi_{i}(j h)=\delta_{i j},
$$

where $\delta$ denotes the Kronecker delta function. By generalizing the definition of onedimensional HFs, 2DHFs can be defined as follows

$$
\Phi_{i, j}(x, y)=\Phi_{i}(x) \Phi_{j}(x), \quad i, j=0,1, \ldots, n .
$$


By substituting (3.1) and (3.2), we have $\Phi_{i, j}(k h, l h)=\delta_{j l} \delta_{i k}$. Now, for the 2DHFs, we have

$$
\phi_{i, j}(x, y) \phi_{k, l}(x, y)=0, \quad|i-j| \geq 2 \text { or }|j-l| \geq 2
$$

and

$$
\sum_{i=0}^{n} \sum_{j=0}^{n} \phi_{i, j}(x, y)=1 .
$$

An arbitrary function $U(x, y)$ can be expanded in vector form as:

$$
U(x, y) \simeq U^{T} \Phi(x, y)=\Phi^{T}(x, y) U
$$

where $U=\left[u_{0}, u_{1}, \ldots, u_{n}\right]^{T}$,

$$
\Phi(x, y)=\left[\phi_{0,0}(x, y), \ldots, \phi_{0, m}(x, y), \phi_{1,0}(x, y), \ldots, \phi_{1,0}(x, y)\right]^{T}
$$

and $u_{i, j}=u(i h, j h), i, j=0,1, \ldots, n$. The positive integer powers of $u(x, y)$ may be approximated by HFs as $[u(x, y)]^{P} \simeq C_{P}^{T} \cdot \Phi(x, y)$. Now, let $k(x, y, s, t)$ be an arbitrary function of two variables defined on $L^{2}([0,1] \times[0,1])$. It can be expanded by HFs as: $k(x, y, s, t) \simeq \Phi^{T}(x, y) K \Phi(s, t)$, where $\Phi(x, y)$ and $\Phi(s, t)$ are 2DHFs vectors of dimention $(n+1)^{2}$, and $K$ is 2DHFs coefficients matrix of dimention $\left(n_{1}+1\right)^{2} \times(n+1)^{2}$ with entries $a_{i j}, i=0,1, \ldots, n_{1}, j=0,1, \ldots, n_{2}$, as $a_{i j}=k(i h, j h)$. In this paper, for convenience, we put $n_{1}=n_{2}=n$. Moreover, from (3.3) follows:

$$
\begin{aligned}
& \Phi(x, y) \Phi^{T}(x, y) \\
& =\left(\begin{array}{ccccc}
\phi_{0}^{2}(x) & \phi_{0}(x) \phi_{1}(x) & & & \\
\phi_{0}(x) \phi_{1}(x) & \phi_{1}^{2}(x) & \phi_{1}(x) \phi_{2}(x) & & \\
& \ddots & \ddots & \ddots & \\
& \ddots & \ddots & \ddots & \\
& & \ddots & \ddots & \phi_{n-1}(x) \phi_{n}(x) \\
& & & \phi_{n-1}(x) \phi_{n}(x) & \phi_{n}^{2}(x)
\end{array}\right) \\
& \otimes\left(\begin{array}{ccccc}
\phi_{0}^{2}(x) & \phi_{0}(x) \phi_{1}(x) & & & \\
\phi_{0}(x) \phi_{1}(x) & \phi_{1}^{2}(x) & \phi_{1}(x) \phi_{2}(x) & & \\
& \ddots & \ddots & \ddots & \\
& \ddots & \ddots & \ddots & \\
& & \ddots & \ddots & \phi_{n-1}(x) \phi_{n}(x) \\
& & & \phi_{n-1}(x) \phi_{n}(x) & \phi_{n}^{2}(x)
\end{array}\right)
\end{aligned}
$$

and

$$
P_{1}=\int_{0}^{1} \int_{0}^{1} \Phi(x, y) \Phi^{T}(x, y) d x d y=\Upsilon_{1} \otimes \Upsilon_{1}
$$


where $P_{1}$ is the following $(n+1) \times(n+1)$ matrix

$$
P_{1}=\frac{h}{6}\left(\begin{array}{ccccc}
2 & 1 & & & \\
1 & 4 & 1 & & \\
& \ddots & \ddots & \ddots & \\
& & \ddots & \ddots & \ddots \\
& & 1 & 4 & 1 \\
& & & 1 & 2
\end{array}\right)
$$

By considering (3.1), and expanding entries of $\Phi(x, y) \Phi^{T}(x, y)$ by 2DHFs, we have $\Phi(x, y) \Phi^{T}(x, y) \simeq \operatorname{diag}(\Phi(x, y))$. Now, suppose that $\Lambda$ is a vector $(n+1)^{2}$. We obtain

$$
\Phi(x, y) \Phi^{T}(x, y) \Lambda \simeq \tilde{\Lambda} \Phi(x, y),
$$

where $\tilde{\Lambda}=\operatorname{diag}(\Lambda)$ is an $(n+1)^{2} \times(n+1)^{2}$-diagonal matrix. Furthermore, if $A$ is an $(n+1)^{2} \times(n+1)^{2}$-matrix, we have

$$
\Phi^{T}(x, y) A \Phi(x, y) \simeq \Phi^{T}(x, y) \hat{A},
$$

where $\hat{A}$ is an $(n+1)^{2}$-vector with elements equal to diagonal entries of matrix $A$. Now, we have

$$
\begin{aligned}
\int_{0}^{y} \int_{0}^{x} \Phi(s, t) d y d t & =\int_{0}^{y} \int_{0}^{x} \Phi(s) \otimes \Phi(t) d s d t=\left(\int_{0}^{y} \Phi(s) d s\right) \otimes\left(\int_{0}^{x} \Phi(t) d t\right) \\
& \simeq\left(\Upsilon_{1} \Phi(x)\right) \otimes\left(\Upsilon_{2} \Phi(y)\right)=\left(\Upsilon_{1} \otimes \Upsilon_{2}\right) \Phi(x, y)=P_{2} \Phi(x, y),
\end{aligned}
$$

where $P_{2}$ is the following $(n+1) \times(n+1)$ matrix

$$
P_{2}=\frac{h}{2}\left(\begin{array}{cccccc}
0 & 1 & 1 & 1 & \cdots & 1 \\
0 & 1 & 2 & 2 & \cdots & 2 \\
0 & 0 & 1 & 2 & \cdots & 2 \\
\vdots & \vdots & \vdots & \vdots & \ddots & \vdots \\
0 & 0 & 0 & 0 & \cdots & 1
\end{array}\right) .
$$

3.1. Operational matrix of the fractional order integration (OMFI). Our goal is to get, to derive the Hat OMFI. For this purpose, Block pulse fractional matrix for the one-dimensional case is presented as follows:

$$
\left(I^{\alpha} f\right)(t)=\frac{1}{\Gamma(\alpha)} \int_{0}^{t}(t-\tau)^{\alpha-1} b(\tau) d \tau=F^{\alpha} b(t),
$$

where $\alpha \in \mathbb{R}$ is the order of the integration and $\Gamma(\alpha)$ is the Gamma function. Also, we define an m-set of Block Pulse Functions (BPFs) as

$$
b_{i}(x)=\left\{\begin{array}{l}
1, \quad \frac{i}{m} \leq x<\frac{(i+1)}{m}, \\
0, \quad \text { otherwise, }
\end{array}\right.
$$


where $i=0,1,2, \ldots, m-1$. The function $b_{i}(x)$ is disjoint and orthogonal, that is

$$
b_{j}(x) b_{i}(x)= \begin{cases}b_{j}, & j=i \\ 0, & j \neq i\end{cases}
$$

where $F^{\alpha}$ is the $m \times m$ fractional operational matrix of integration of order $\alpha$ for the BPFs (see [16]) where

$$
\begin{aligned}
& \left(I^{\alpha} B_{m}\right)(x) \simeq F^{\alpha} B_{m}(x), \\
& F^{\alpha}=\frac{1}{m^{\alpha}} \frac{1}{\Gamma(\alpha+2)}\left[\begin{array}{cccccc}
1 & \xi_{1} & \xi_{2} & \xi_{3} & \ldots & \xi_{m-1} \\
0 & 1 & \xi_{1} & \xi_{2} & \ldots & \xi_{m-1} \\
0 & 0 & 1 & \xi_{1} & \ldots & \xi_{m-3} \\
\vdots & \vdots & \ddots & \ddots & & \vdots \\
0 & 0 & \ldots & 0 & 1 & \xi_{1} \\
0 & 0 & 0 & \ldots & 0 & 1
\end{array}\right]
\end{aligned}
$$

and $\xi_{k}=(k+1)^{\alpha+1}-2 k^{\alpha+1}+(k-1)^{\alpha+1}$. Our aim is to derive the Hat OMFI. For this purpose, we used the Riemann-Liouville fractional order integration, as following:

$$
\begin{aligned}
\left(I^{\alpha} u\right)(x, y) & =\frac{1}{\Gamma(\alpha) \Gamma(\beta)} \int_{0}^{y} \int_{0}^{x}(y-s)^{\alpha-1}(x-t)^{\beta-1} u(s, t) d s d t \\
& =\frac{1}{\Gamma(\alpha) \Gamma(\beta)} y^{\alpha-1} x^{\beta-1} * u(x, y),
\end{aligned}
$$

where $\alpha, \beta \in \mathbb{R}$ are the order of the integration, $\Gamma(\alpha)$ and $\Gamma(\beta)$ are the Gamma functions and $y^{\alpha-1} * u(x, y), x^{\beta-1} * u(x, y)$ denote the convolution products of $y^{\alpha-1}$, $x^{\beta-1}$ and $u(x, y)$. Now if $u(x, y)$ is expanded in HFs, as shown in (3.4), the RiemannLiouville fractional integration becomes

$$
\left(I^{\alpha} u\right)(x, y)=\frac{1}{\Gamma(\alpha) \Gamma(\beta)} y^{\alpha-1} x^{\beta-1} * u(x, y) \approx C^{T} \frac{1}{\Gamma(\alpha) \Gamma(\beta)} y^{\alpha-1} x^{\beta-1} * \Phi(x, y) .
$$

Thus, if $y^{\alpha-1} * u(x, y)$ and $x^{\beta-1} * u(x, y)$ can be integrated, then by expanding the Hat functions, the Riemann-Liouville fractional order integration solve the HFs. Also, we define an $m$-set of BPF as

$$
b_{i_{1}, i_{2}}(x, y)= \begin{cases}1, & \left(i_{1}-1\right) h_{1} \leqslant x<i_{1} h_{1} \text { and }\left(i_{2}-1\right) h_{2} \leqslant y<i_{2} h_{2}, \\ 0, & \text { otherwise }\end{cases}
$$

where $i=0,1,2, \ldots, m-1$. The function $b_{i, j}(t)$ is disjoint and orthogonal, that is

$$
b_{i_{1}, i_{2}}(x, y) b_{j_{1}, j_{2}}(x, y)= \begin{cases}b_{i_{1}, i_{2}}(x, y), & i_{1}=j_{1} \text { and } i_{2}=j_{2}, \\ 0, & \text { otherwise }\end{cases}
$$

The HFs can be expanded in to $m$-set of BPs functions as

$$
\Phi(x, y)=\Psi_{m \times m} B_{m}(x, y),
$$

where $B_{m}(x)=\left(b_{0}(x), b_{1}(x), \ldots, b_{i}(x), \ldots, b_{m-1}(x)\right)^{T}($ see $[24,25])$ and $\Psi$ is an $M N \times$ $M N$ product operational matrix. Next, we derive the Hat OMFI. We have the two 
dimensional BPFs operational matrix of fractional integration as:

$$
\frac{1}{\Gamma(\alpha) \Gamma(\beta)} \int_{0}^{y} \int_{0}^{x}(y-s)^{\alpha-1}(x-t)^{\beta-1} U(s, t) d s d t=F^{\alpha, \beta} U(x, y)
$$

where

$$
\begin{aligned}
F^{\alpha, \beta}= & \frac{1}{m^{\alpha} m^{\beta}} \frac{1}{\Gamma(\alpha+2) \Gamma(\beta+2)} \\
& \times\left[\begin{array}{cccccc}
1 & \xi_{1} & \xi_{2} & \xi_{3} & \ldots & \xi_{m-1} \\
0 & 1 & \xi_{1} & \xi_{2} & \ldots & \xi_{m-1} \\
0 & 0 & 1 & \xi_{1} & \ldots & \xi_{m-3} \\
\vdots & \vdots & \ddots & \ddots & & \vdots \\
0 & 0 & \ldots & 0 & 1 & \xi_{1} \\
0 & 0 & 0 & \ldots & 0 & 1
\end{array}\right] \otimes\left[\begin{array}{cccccc}
1 & \eta_{1} & \eta_{2} & \eta_{3} & \ldots & \eta_{m-1} \\
0 & 1 & \eta_{1} & \eta_{2} & \ldots & \eta_{m-1} \\
0 & 0 & 1 & \eta_{1} & \ldots & \eta_{m-3} \\
\vdots & \vdots & \ddots & \ddots & & \vdots \\
0 & 0 & \ldots & 0 & 1 & \eta_{1} \\
0 & 0 & 0 & \ldots & 0 & 1
\end{array}\right]
\end{aligned}
$$

$\xi_{k}=(k+1)^{\alpha+1}-2 k^{\alpha+1}+(k-1)^{\alpha+1}$ and $\eta_{k}=(k+1)^{\beta+1}-2 k^{\beta+1}+(k-1)^{\beta+1}$.

Fractional integration of the BPFs is given as the following

$$
\left(I^{\alpha, \beta} B_{m}\right)(x, y) \approx F^{\alpha, \beta} B_{m}(x, y) .
$$

Now, we derive the HFs operational matrix of the fractional order integration. Let

$$
\left(I^{\alpha, \beta} \Phi\right)(x, y) \approx P_{m \times m}^{\alpha, \beta} \Phi(x, y)
$$

where matrix $P_{m \times m}^{\alpha, \beta}$ is called the Hat functions OMFI. Using (3.7) and (3.8), we have

$$
\left(I^{\alpha, \beta} \Phi\right)(x, y) \approx\left(I^{\alpha, \beta} \Psi_{m \times m} B_{m}\right)(x, y)=\Psi_{m \times m}\left(I^{\alpha} B_{m}\right)(x, y) \approx \Psi_{m \times m} F^{\alpha, \beta} B_{m}(x, y) .
$$

By (3.9) and (3.10) we get

$$
P_{m \times m}^{\alpha, \beta} \Phi(x, t)=\Psi_{m \times m} F^{\alpha, \beta} B_{m}(x, y)=\Psi_{m \times m} F^{\alpha, \beta} \Phi_{m \times m} \Psi_{m \times m}^{-1} .
$$

Then, the Hat functions OMFI $P_{m \times m}^{\alpha, \beta}$ is given by

$$
P_{m \times m}^{\alpha, \beta}=\Psi_{m \times m} F^{\alpha, \beta} \Psi_{m \times m}^{-1} .
$$

\section{Applying the Method}

In this section, 2DHFs fractional operational matrix are applied to solving (1.1). Now, let

$$
D_{*}^{\alpha} u(x, y) \simeq C^{T} \Phi(x, y) .
$$

By using (4.1) and (3.9) and Lemma 2.1, we have

$$
u(x, y)=C^{T} P_{m \times m}^{\alpha} \Phi(x, y)+\sum_{k=0}^{m-1} \frac{\partial^{k} u\left(0^{+}, y\right)}{\partial x^{k}} \frac{x^{k}}{k !}, \quad x>0 .
$$

So, by replacing the supplementary initial conditions (1.2), in the above summation in the above equations and approximating it by Hat functions, we have

$$
u(x, y) \cong\left(C^{T} P_{m \times m}^{\alpha}+C_{p}^{T}\right) \Phi(x, y),
$$


where $C_{p}$ is a column $m$-vector. Define $e=\left[e_{0}, e_{1}, \ldots, e_{m-1}\right]=\left(C^{T} P_{m \times m}^{\alpha}+C_{p}^{T}\right)$, so, $u(x, y) \cong e \Phi(x, y)$. We could easily check out the correctness of the expression with induction $[u(x, y)]^{q} \cong\left[e_{0}^{q}, e_{1}^{q}, \ldots, e_{m-1}^{q}\right] \Phi(x, y)=e_{q} \Phi_{m \times m}$, where $\tilde{e}_{q}=\left[e_{0}^{q}, e_{1}^{q}, \ldots, e_{m-1}^{q}\right]$. The function $u(x, y), k(x, y, s, t)$ and $f(x, y)$ can be approximated by

$$
\begin{aligned}
u(x, y) & =U^{T} \Phi(x, y)=U \Phi^{T}(x, y), \\
F(x, y) & =F^{T} \Phi(x, y)=F \Phi^{T}(x, y), \\
{[u(x, y)]^{p} } & =\Phi^{T}(x, y) C_{p}, \\
k(x, y, s, t) & =\Phi^{T}(x, y) \cdot K \cdot \Phi(s, t) .
\end{aligned}
$$

Now, with substituting (4.2) in (1.1), we have

$$
D_{x}^{\alpha} u(x, y)=\frac{1}{\Gamma(\alpha) \Gamma(\beta)} \int_{0}^{y} \int_{0}^{x}(y-s)^{\alpha-1}(x-t)^{(\beta-1)} G(x, y, s, t, u(s, t)) d s d t+f(x, y) .
$$

Using (3.5), (3.6), (3.9), and (3.11), we have

$$
\begin{aligned}
& C \Phi^{T}(x, y) \\
= & \frac{1}{\Gamma(\alpha) \Gamma(\beta)} \int_{0}^{y} \int_{0}^{x}(y-s)^{\alpha-1}(x-t)^{(\beta-1)} k(x, y, s, t)[u(s, t)]^{p} d s d t+F \Phi^{T}(x, y) \\
= & \frac{1}{\Gamma(\alpha) \Gamma(\beta)} \int_{0}^{y} \int_{0}^{x}(y-s)^{\alpha-1}(x-t)^{(\beta-1)} \Phi^{T}(x, t) K \Phi(s, t) \Phi^{T}(x, y) C_{p} d s d t+F \Phi^{T}(x, y) \\
= & \Phi^{T}(x, y) K \tilde{C}_{p} \frac{1}{\Gamma(\alpha) \Gamma(\beta)} \int_{0}^{y} \int_{0}^{x}(y-s)^{\alpha-1}(x-t)^{(\beta-1)} \Phi(s, t) d s d t+F \Phi^{T}(x, y) \\
= & \Phi^{T}(x, y) K \tilde{C}_{p} P_{m \times m}^{\alpha, \beta} \Phi(x, y)=\left(\widehat{K \tilde{c} p P_{m \times m}^{\alpha, \beta}}\right)^{T} \cdot \Phi(x, y)+F \Phi^{T}(x, y) \\
= & \left(\widehat{K \tilde{c} p P_{m \times m}^{\alpha, \beta}}\right) \cdot \Phi^{T}(x, y)+F \Phi^{T}(x, y) .
\end{aligned}
$$

Set

so,

$$
B=\left(\widehat{K \tilde{c} p P_{m \times m}^{\alpha, \beta}}\right)
$$

$$
C \Phi^{T}(x, y)=B \Phi^{T}(x, y)+F \Phi^{T}(x, y)
$$

hence, we have

$$
C=B+F
$$

which is a system of algebraic equations. By solving this system, we can obtain the approximate solution of (1.1) according to (4.3).

\section{Convergence And Error Analysis}

In this section, we obtain an error bound for the approximate solution, then from which we conclude convergence of the method. We define the error function as

$$
e_{n}(x, y)=u(x, y)-\hat{u}(x, y)
$$


where $u(x, y)$ and $\hat{u}(x, y)$ denote the exact and approximate solutions, respectively.

Theorem 5.1. Suppose $u(x, y) \in I$ and $e_{n}(x, y)=u(x, y)-u_{n}(x, y),(x, y) \in I=$ $[0, T) \times[0, T)$, where $u_{n}(x, y)=\sum_{i=0}^{n} u(i h, j h) \phi_{i, j}(x, y)$ is the generalized hat function expansion of $u(x, y)$. Then, we have

$$
\left\|e_{n}(x, y)\right\| \leq \frac{T^{2}}{2 n^{2}}\left\|u^{\prime \prime}(x, y)\right\|
$$

and so the convergence is of order two, that is $\left\|e_{n}(x, y)\right\|=O\left(\frac{1}{n^{2}}\right)$.

Proof. See [17].

Theorem 5.2. Suppose $u(x, y)$ as an exact solution of fractional integral (1.1) and $\hat{u}(x, y)$ show the approximate solution by Hat functions. If $\left|(x-s)^{\alpha-1}(y-t)^{\beta-1} k(x, y, s, t)\right|<N, u(x, y)$ and $k(x, y, s, t)$ are continuous functions and also, $G(u)=(u(x, t))^{p}$ satisfies Lipschitz condition $|G(u)-G(\hat{u})| \leq L|u-\hat{u}|$, then

$$
\|u-\hat{u}\|=\sup _{0 \leq x, y \leq 1}|u(x, y)-\hat{u}(x, y)|=O\left(\frac{1}{n^{2}}\right) .
$$

Proof. We have

$$
\begin{aligned}
& |u(x, y)-\hat{u}(x, y)| \\
= & \left|\frac{1}{\Gamma(\alpha) \Gamma(\beta)} \int_{0}^{y} \int_{0}^{x}(y-s)^{\alpha-1}(x-t)^{\beta-1} k(x, y, s, t)(u(s, t)-\hat{u}(s, t)) d t d s\right| \\
\leq & \frac{1}{\Gamma(\alpha) \Gamma(\beta)} \int_{0}^{x} \int_{0}^{y}\left|(x-s)^{\alpha-1}(y-t)^{\beta-1} k(x, y, s, t)(u(s, t)-\hat{u}(s, t))\right| d s d t \\
\leq & \frac{1}{\Gamma(\alpha) \Gamma(\beta)} \int_{0}^{y} \int_{0}^{x}\left|(y-s)^{\alpha-1}(x-t)^{\beta-1} k(x, y, s, t)\right||(u(s, t)-\hat{u}(s, t))| d s d t \\
\leq & \frac{N}{\Gamma(\alpha) \Gamma(\beta)} \int_{0}^{y} \int_{0}^{x}|(u(s, t)-\hat{u}(s, t))| d s d t .
\end{aligned}
$$

From (5.1), we conclude that

$$
|u(x, y)-\hat{u}(x, y)| \leq \frac{N L T^{2} x y}{2 n^{2} \Gamma(\alpha) \Gamma(\beta)} \leq \frac{N L T^{2}}{2 n^{2} \Gamma(\alpha) \Gamma(\beta)} .
$$

This completes the proof.

Theorem 5.3. The solving systems of partial 2 DFVIE by using $2 D$-HFs converge if $0<\theta<1$, where $\theta=\frac{N L T^{2}}{2 n^{2} \Gamma(\alpha) \Gamma(\beta)}$.

Proof. If we assume $G(u)=D_{x}^{\alpha} u(x, y)$, we have $\left\|G(u)-G\left(u_{m}\right)\right\|_{\infty} \leq\left\|u-u_{m}\right\|_{\infty}$. From Theorem 5.2, we have

$$
\left\|G(u)-G\left(u_{m}\right)\right\|_{\infty} \leq \frac{N L T^{2}}{2 n^{2} \Gamma(\alpha) \Gamma(\beta)}\left\|u-u_{m}\right\|_{\infty} .
$$


Inequality (5.2) implies that if $0<\theta<1$, then we have $\lim _{m \rightarrow \infty}\left\|G(u)-G\left(u_{m}\right)\right\|_{\infty}=0$ and $\lim _{m \rightarrow \infty}\left\|u-u_{m}\right\|_{\infty}=0$.

\section{Numerical Examples}

To illustrate the effectiveness of the proposed method in the present paper, some test examples are carried out in this section.

Example 6.1. Consider the fractional partial volterra integro-differential equation [19]

$$
D_{x}^{0.75} u(x, y)=\int_{0}^{y} \int_{0}^{x}(y+t) u(s, t) d s d t=\frac{6.4}{\Gamma(0.25)} y x^{5 / 4}-\frac{5}{18} x^{3} y^{3},
$$

where the exact solution is known and it is given by $u(x, y)=x^{2} y$, for $x, y \in[0,1]$ and with supplementary condition $u(0, y)=0$. Numerical results are presented in Table 1.

TABLE 1. The absolute errors for Example 1.

\begin{tabular}{lcccc}
\hline & $m=n=4$ & $m=n=4$ & $m=n=5$ & $m=n=5$ \\
\hline$(x, y)$ & $u_{2 D L W s}[19]$ & $u_{2 D H F s}$ & $u_{2 D L W s}[19]$ & $u_{2 D H F s}$ \\
\hline$(0.0,0.7)$ & $0.1404 \times 10^{-2}$ & $0.1404 \times 10^{-2}$ & $0.3508 \times 10^{-3}$ & $0.2327 \times 10^{-3}$ \\
$(0.1,0.3)$ & $0.1636 \times 10^{-3}$ & $0.2584 \times 10^{-2}$ & $0.1342 \times 10^{-3}$ & $0.4158 \times 10^{-3}$ \\
$(0.3,0.8)$ & $0.1456 \times 10^{-2}$ & $0.3651 \times 10^{-3}$ & $0.8962 \times 10^{-3}$ & $0.1001 \times 10^{-4}$ \\
$(0.4,0.2)$ & $0.1087 \times 10^{-3}$ & $0.6521 \times 10^{-3}$ & $0.2700 \times 10^{-4}$ & $0.5057 \times 10^{-4}$ \\
$(0.6,0.6)$ & $0.3248 \times 10^{-3}$ & $0.1421 \times 10^{-3}$ & $0.6759 \times 10^{-3}$ & $0.5884 \times 10^{-4}$ \\
$(0.7,0.5)$ & $0.8878 \times 10^{-3}$ & $0.6250 \times 10^{-3}$ & $0.5285 \times 10^{-4}$ & $0.1019 \times 10^{-4}$ \\
$(0.8,0.4)$ & $0.7061 \times 10^{-3}$ & $0.7247 \times 10^{-3}$ & $0.4090 \times 10^{-4}$ & $0.1018 \times 10^{-4}$ \\
$(0.9,0.9)$ & $0.5898 \times 10^{-3}$ & $0.1997 \times 10^{-3}$ & $0.1974 \times 10^{-3}$ & $0.4108 \times 10^{-4}$ \\
\hline
\end{tabular}

Example 6.2. Consider the linear two-dimensional fractional integro-differential equation [19]

$$
D_{x}^{0.5} u(x, y)=\int_{0}^{y} \int_{0}^{x}\left(x^{2} y+s\right) u(s, t) d s d t=4 y \sqrt{\frac{x}{\pi}}-\frac{1}{2} x^{4} y^{3}-\frac{1}{3} x^{3} y^{2},
$$

where the exact solution is known and given by $u(x, y)=2 x y$, for $x, y \in[0,1]$ and with supplementary condition $u(0, y)=0$. Numerical results are presented in the Table 2.

Example 6.3. Consider the linear two-dimensional fractional integro-differential equation [19]

$$
D_{x}^{0.5} u(x, y)=\int_{0}^{y} \int_{0}^{x}(x \cos (s)+y t) u(s, t) d s d t=f(x, y)
$$


TABLE 2. The absolute errors for Example 2.

\begin{tabular}{lcccc}
\hline & $m=n=4$ & $m=n=4$ & $m=n=5$ & $m=n=5$ \\
\hline$(x, y)$ & $u_{2 D L W s}[19]$ & $u_{2 D H F s}$ & $u_{2 D L W s}[19]$ & $u_{2 D H F s}$ \\
\hline$(0.1,0.8)$ & $0.1173 \times 10^{-3}$ & $0.1853 \times 10^{-3}$ & $0.1250 \times 10^{-3}$ & $0.4141 \times 10^{-3}$ \\
$(0.2,0.6)$ & $0.1805 \times 10^{-3}$ & $0.9461 \times 10^{-3}$ & $0.2751 \times 10^{-4}$ & $0.4258 \times 10^{-3}$ \\
$(0.3,0.8)$ & $0.9276 \times 10^{-4}$ & $0.9276 \times 10^{-4}$ & $0.1189 \times 10^{-4}$ & $0.1104 \times 10^{-4}$ \\
$(0.4,0.6)$ & $0.2710 \times 10^{-4}$ & $0.3621 \times 10^{-4}$ & $0.1395 \times 10^{-5}$ & $0.1245 \times 10^{-5}$ \\
$(0.5,0.5)$ & $0.7309 \times 10^{-5}$ & $0.1001 \times 10^{-4}$ & $0.4065 \times 10^{-5}$ & $0.7412 \times 10^{-5}$ \\
$(0.6,0.5)$ & $0.3884 \times 10^{-4}$ & $0.3621 \times 10^{-4}$ & $0.1174 \times 10^{-4}$ & $0.3241 \times 10^{-5}$ \\
$(0.7,0.3)$ & $0.3548 \times 10^{-4}$ & $0.5200 \times 10^{-3}$ & $0.9798 \times 10^{-5}$ & $0.4142 \times 10^{-4}$ \\
$(0.8,0.4)$ & $0.9069 \times 10^{-4}$ & $0.3247 \times 10^{-4}$ & $0.2406 \times 10^{-4}$ & $0.3258 \times 10^{-4}$ \\
$(0.9,0.9)$ & $0.6179 \times 10^{-3}$ & $0.1657 \times 10^{-3}$ & $0.1607 \times 10^{-3}$ & $0.4741 \times 10^{-4}$ \\
\hline
\end{tabular}

where

$$
\begin{aligned}
f(x, y)= & \frac{2 \sin (y) \sqrt{x}}{\sqrt{0.5}}+x \cos (x)-x^{2} \sin (x)-x \cos (y)+x \cos (x) \cos (y) \\
& +x^{2} \sin (x) \cos (y)-\frac{1}{2} x^{2} y \sin (y)+\frac{1}{2} x^{2} y^{2} \cos (y),
\end{aligned}
$$

where the exact solution is known and given by $u(x, y)=x \sin (y)$, for $x, y \in[0,1]$ and with supplementary condition $u(0, y)=0$. Numerical results are presented in the Table 3.

TABLE 3. The absolute errors for Example 3.

\begin{tabular}{lcccc}
\hline & $m=n=3$ & $m=n=3$ & $m=n=4$ & $m=n=4$ \\
\hline$(x, y)$ & $u_{2 D L W s}[19]$ & $u_{2 D H F s}$ & $u_{2 D L W s}[19]$ & $u_{2 D H F s}$ \\
\hline$(0.1,0.1)$ & $0.1599 \times 10^{-3}$ & $0.2514 \times 10^{-2}$ & $0.5398 \times 10^{-4}$ & $0.9841 \times 10^{-3}$ \\
$(0.2,0.2)$ & $0.2155 \times 10^{-3}$ & $0.6251 \times 10^{-3}$ & $0.5185 \times 10^{-4}$ & $0.4625 \times 10^{-4}$ \\
$(0.3,0.3)$ & $0.1566 \times 10^{-3}$ & $0.5210 \times 10^{-3}$ & $0.6503 \times 10^{-4}$ & $0.1984 \times 10^{-4}$ \\
$(0.4,0.4)$ & $0.2122 \times 10^{-3}$ & $0.9654 \times 10^{-3}$ & $0.7688 \times 10^{-4}$ & $0.1962 \times 10^{-4}$ \\
$(0.5,0.5)$ & $0.2477 \times 10^{-3}$ & $0.2014 \times 10^{-3}$ & $0.8809 \times 10^{-4}$ & $0.7620 \times 10^{-4}$ \\
$(0.6,0.6)$ & $0.2971 \times 10^{-3}$ & $0.6521 \times 10^{-3}$ & $0.9899 \times 10^{-4}$ & $0.3021 \times 10^{-4}$ \\
$(0.7,0.7)$ & $0.3662 \times 10^{-3}$ & $0.6214 \times 10^{-3}$ & $0.1226 \times 10^{-3}$ & $0.4142 \times 10^{-4}$ \\
$(0.8,0.8)$ & $0.4738 \times 10^{-3}$ & $0.2147 \times 10^{-3}$ & $0.1599 \times 10^{-3}$ & $0.3108 \times 10^{-4}$ \\
$(0.9,0.9)$ & $0.6344 \times 10^{-3}$ & $0.9651 \times 10^{-3}$ & $0.2246 \times 10^{-3}$ & $0.4748 \times 10^{-3}$ \\
\hline
\end{tabular}

Example 6.4. Consider the two-dimensional fractional Volterra integral equation [1]

$$
u(x, y)-\frac{1}{\Gamma\left(\frac{7}{2}\right) \Gamma\left(\frac{5}{2}\right)} \int_{0}^{y} \int_{0}^{x}(y-s)^{\frac{5}{2}}(x-t)^{\frac{3}{2}}\left(y^{2}+s\right) e^{-t} u(s, t) d s d t=f(x, y),
$$

where

$$
f(x, y)=x^{2} e^{y}-\frac{1024 x^{\frac{11}{2}} y^{\frac{5}{2}}\left(6 x+13 y^{2}\right)}{2027025 \pi},
$$


where the exact solution is known and it is given by $u(x, y)=x^{2} e^{y}$. To solve this equation, we implement the HFs method for $\alpha=\frac{7}{2}$ and $\beta=\frac{5}{2}$. Numerical results are presented in Table 4 and Figure 1.

TABLE 4. The absolute errors for Example 4.

\begin{tabular}{lcccc}
\hline & $m=n=2$ & $m=n=2$ & $m=n=4$ & $m=n=4$ \\
\hline$x=y$ & $u_{2 D B P O M}[1]$ & $u_{2 D H F s}$ & $u_{2 D B P O M}[1]$ & $u_{2 D H F s}$ \\
\hline 0.0 & $2.090 \times 10^{-4}$ & $2.125 \times 10^{-4}$ & $4.086 \times 10^{-4}$ & $5.237 \times 10^{-5}$ \\
0.1 & $2.532 \times 10^{-4}$ & $2.635 \times 10^{-4}$ & $4.181 \times 10^{-4}$ & $4.258 \times 10^{-5}$ \\
0.2 & $6.967 \times 10^{-5}$ & $5.689 \times 10^{-4}$ & $4.471 \times 10^{-4}$ & $4.125 \times 10^{-4}$ \\
0.3 & $2.602 \times 10^{-4}$ & $3.070 \times 10^{-4}$ & $4.970 \times 10^{-4}$ & $4.157 \times 10^{-4}$ \\
0.4 & $3.346 \times 10^{-4}$ & $4.325 \times 10^{-4}$ & $5.656 \times 10^{-4}$ & $4.984 \times 10^{-4}$ \\
0.5 & $2.778 \times 10^{-4}$ & $3.215 \times 10^{-3}$ & $6.474 \times 10^{-4}$ & $6.259 \times 10^{-4}$ \\
0.6 & $1.701 \times 10^{-3}$ & $2.587 \times 10^{-3}$ & $7.316 \times 10^{-4}$ & $7.147 \times 10^{-4}$ \\
0.7 & $2.090 \times 10^{-3}$ & $2.090 \times 10^{-3}$ & $7.817 \times 10^{-4}$ & $7.548 \times 10^{-4}$ \\
0.8 & $3.542 \times 10^{-3}$ & $3.985 \times 10^{-3}$ & $6.788 \times 10^{-4}$ & $7.214 \times 10^{-4}$ \\
0.9 & $1.137 \times 10^{-3}$ & $2.087 \times 10^{-3}$ & $1.004 \times 10^{-4}$ & $2.587 \times 10^{-4}$ \\
\hline
\end{tabular}

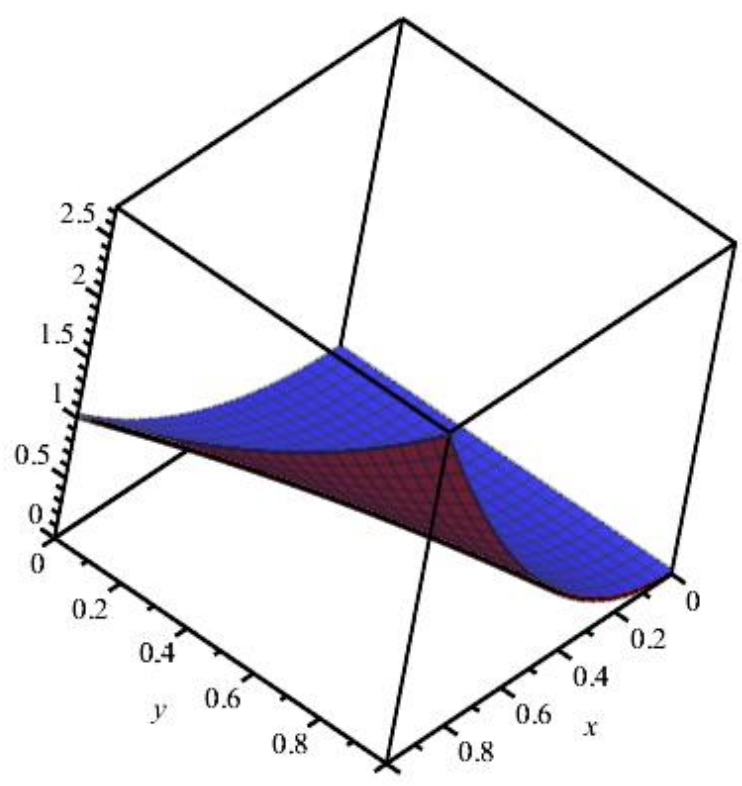

FiguRE 1. Exact and approximation solutions of Example 4.

Example 6.5. Consider the two-dimensional nonlinear fractional Volterra equation [20]

$$
u(x, y)-\frac{1}{\Gamma\left(\frac{3}{2}\right) \Gamma\left(\frac{5}{2}\right)} \int_{0}^{y} \int_{0}^{x}(y-s)^{\frac{1}{2}}(x-t)^{\frac{3}{2}} \sqrt{x y t}[u(s, t)]^{2} d s d t=f(x, y),
$$


where

$$
f(x, y)=\sqrt{y}\left(\frac{-1}{180} x^{3} y^{\frac{7}{2}}+\sqrt{\frac{x}{3}}\right) .
$$

The exact solution is known and it is given by $u(x, y)=\frac{\sqrt{3 x y}}{3}$. This example has been solved, for $\alpha=\frac{3}{2}$ and $\beta=\frac{5}{2}$. Numerical results for this a solution are presented in Table 5 and Figure 2.

TABLE 5. The numerical results for Example 5.

\begin{tabular}{lccc}
\hline & Exact solution & $m=32$ & $m=32$ \\
\hline$x=y$ & & $u_{2 D B P F s}[20]$ & $u_{2 D H F s}$ \\
\hline 0.0 & 0 & 0.009386 & 0.002541 \\
0.1 & 0.05773 & 0.042121 & 0.042541 \\
0.2 & 0.11547 & 0.124282 & 0.138744 \\
0.3 & 0.17323 & 0.156905 & 0.144871 \\
0.4 & 0.23094 & 0.239179 & 0.235487 \\
0.5 & 0.28867 & 0.274574 & 0.275487 \\
0.6 & 0.34641 & 0.354075 & 0.344872 \\
0.7 & 0.40414 & 0.389848 & 0.404151 \\
0.8 & 0.46188 & 0.468971 & 0.469874 \\
0.9 & 0.50702 & 0.507021 & 0.507210 \\
\hline
\end{tabular}

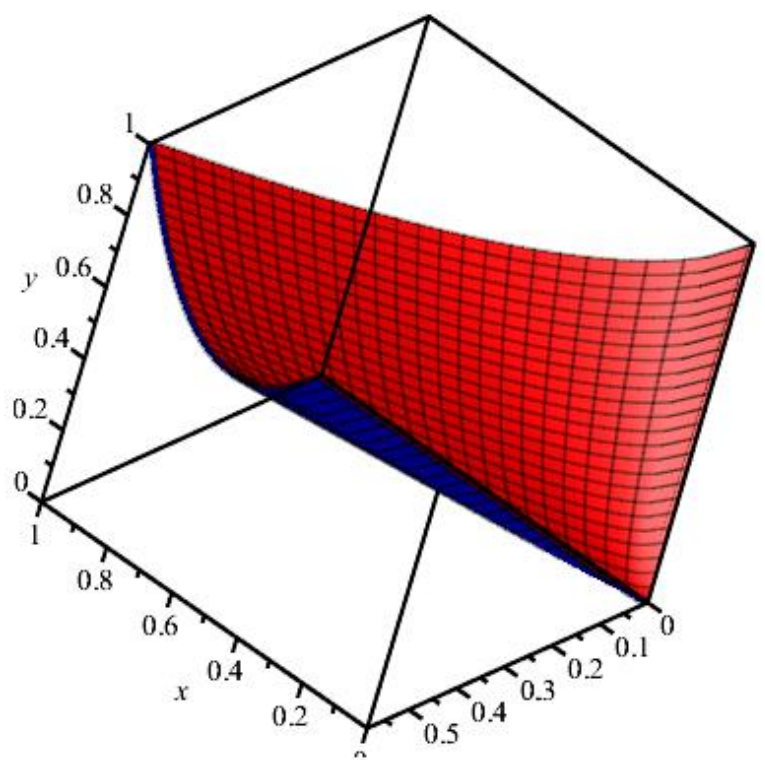

Figure 2. Comparison the exact solution and the presented method for Example 5. 


\section{Conclusion}

In this paper, a Hat operational matrix of fractional order integration is obtained and it is used to solve the two-dimensional nonlinear fractional Volterra integro-differential equations. By properties of 2DHFs and using of operational matrices the possibility of reducing these equations to a system of algebraic equations are provided. Moreover, a general procedure of forming this matrix $P_{m \times m}^{\alpha, \beta}$ is summarized. For more investigation, some examples are presented. As the numerical results showed, the proposed method is an accurate and effective method for solving a fractional two-dimensional integral equation.

\section{REFERENCES}

[1] M. Asgari and R. Ezzati, Using operational matrix of two-dimensional Bernstein polynomials for solving two-dimensional integral equations of fractional order, Appl. Math. Comput. 307 (2017), 290-298.

[2] S. Abbasa and M. Benchohra, Fractional order integral equations of two independent variables, Appl. Math. Comput. 227 (2014), 755-761.

[3] A. Arikoglu and I. Ozkol, Solution of fractional integro-differential equations by using fractional differential transform method, Chaos Solitons Fractals 40 (2009), 521-529.

[4] N. Aghazadeh and A. A. Khajehnasiri, Solving nonlinear two-dimensional Volterra integrodifferential equations by block-pulse functions, Mathematical Sciences 7 (2013), 1-6.

[5] E. Babolian and M. Mordad, A numerical method for solving systems of linear and nonlinear integral equations of the second kind by hat basis functions, Comput. Math. Appl. 62 (2011), $187-198$.

[6] E. H. Doha, A. H. Bhrawy and S. S. Ezz-Eldien, A Chebyshev spectral method based on operational matrix for initial and boundary value problems of fractional order, Comput. Math. Appl. 62 (2011), 2364-2373.

[7] A. Ebadian and A. A. Khajehnasiri, Block-pulse functions and their applications to solving systems of higher-order nonlinear Volterra integro-differential equations, Electron. J. Differ. Equ. 54 (2014), 1-9.

[8] A. Ebadian, H. Rahmani Fazli and A. A. Khajehnasiri, Solution of nonlinear fractional diffusionwave equation by traingular functions, SeMA Journal 72 (2015), 37-46.

[9] L. Gaul, P. Klein and S. Kempfle, Damping description involving fractional operators, Mechanical Systems and Signal Processing 5 (1991), 81-88.

[10] W. G. Glockle and T. F. Nonnenmacher, A fractional calculus approach of self-similar protein dynamics, Biophysical Journal 68 (1995), 46-53.

[11] M. H. Heydari, M. R. Hooshmandasl, F. M. Maalek Ghaini and C. Cattani, A computational method for solving stochastic Ito-Volterra integral equations based on stochastic operational matrix for generalized hat basis functions, J. Comput. Phys. 270 (2014), 402-415.

[12] C. Hwang and Y. P. Shih, Parameter identification via Laguerre polynomials, Internat. J. Systems Sci. 13 (1982), 209-217.

[13] M. H. Heydari, M. R. Hooshmandasl, F. M. Maalek Ghaini and C. Cattani, An efficient computational method for solving nonlinear stochastic Ito integral equations: application for stochastic problems in physics, J. Comput. Phys. 283 (2015), 148-168.

[14] D. Jabari Sabeg, R. Ezzati and K. Maleknejad, A new operational matrix for solving twodimensional nonlinear integral equations of fractional order, Cogent Math. Stat. 4 (2017), 1-11. 
[15] A. A. Khajehnasiri, Numerical Solution of Nonlinear 2D Volterra-Fredholm Integro-Differential Equations by Two-Dimensional Triangular Function, 2 Int. J. Appl. Comput. Math. (2016), $575-591$.

[16] A. Kilicman and Z. A. Al Zhour, Kronecker operational matrices for fractional calculus and some applications, Commun. Appl. Math. Comput. 187 (2007), 250-265.

[17] F. Mirzaee and E. Hadadiyan, Application of two-dimensional hat functions for solving spacetime integral equations, J. Appl. Math. Comput. 4 (2015), 1-34.

[18] S. Momani, and M. A. Noor, Numerical methods for fourth-order fractional integro-differential equations, Appl. Math. Comput. 182 (2006), 754-760.

[19] M. Mojahedfar, A. Tari Marzabad, Solving two-dimensional fractional integro-differential equations by legendre wavelets, Bull. Iranian Math. Soc. 43 (2017), 2419-2435.

[20] S. Najafalizadeh and R. Ezzati, Numerical methods for solving two-dimensional nonlinear integral equations of fractional order by using two-dimensional block pulse operational matrix, Appl. Math. Comput. 280 (2016), 46-56.

[21] P. N. Paraskevopoulos. Legendre series approach to identification and analysis of linear systems, IEEE Trans. Automat. Control 30 (1985), 585-589.

[22] H. Rahmani Fazli, F. Hassani, A. Ebadian and A. A. Khajehnasiri, National economies in state-space of fractional-order financial system, Afr. Mat. 10 (2015), 1-12.

[23] E. A. Rawashdeh, Numerical solution of fractional integro-differential equations by collocation method, Appl. Math. Comput. 176 (2006), 1-6.

[24] H. Saeedi, N. Mollahasani, M. M. Moghadam and G. N. Chuev, An operational haar wavelet method for solving fractional Volterra integral equations, Int. J. Appl. Math. Comput. Sci. 21 (2011), 535-547.

[25] M. Saeedi and M. M. Moghadam, Numerical solution of nonlinear Volterra integro-differential equations of arbitrary order by CAS Wavelets, Commun. Nonlinear Sci. Numer. Simul. 16 (2011), 1216-1226.

[26] A. Saadatmandi and M. Dehghan, A new operational matrix for solving fractional-order differential equations, Comput. Math. Appl. 59 (2010), 1326-1336.

[27] M. P. Tripathi, V. K. Baranwal, R. K. Pandey and O. P. Singh, A new numerical algorithm to solve fractional differential equations based on operational matrix of generalized hat functions, Commun. Nonlinear Sci. Numer. Simul. 18 (2013), 1327-1340.

[28] M. Yi, J. Huang and J. Wei, Block pulse operational matrix method for solving fractional partial differential equation, Appl. Math. Comput. 221 (2013), 121-131.

${ }^{1}$ Department of Mathematics, North Tehran Branch,

ISLAMIC AZAD UNIVERSITY, TEHRAN, IRAN

Email address: a.khajehnasiri@gmail.com

Email address: m-afshar@iau-tnb.ac.ir

${ }^{2}$ Department of Mathematics,

Karaj Branch, Islamic Azad University, KaraJ, Iran

Email address: ezati@kiau.ac.ir

${ }^{*}$ Corresponding AUthor 